\title{
Compact UHF Antenna in Aquatic Environments for Mobile Sporting Applications
}

\author{
Amin M. Abbosh ${ }^{*(1)}$, Daniel James ${ }^{(2,3)}$, and David V. Thiel ${ }^{(2)}$ \\ (1) School of ITEE, The University of Queensland, Australia \\ (2) Centre for Wireless Monitoring and Applications, Griffith University, Australia \\ ${ }^{(3)}$ Centre of Excellence for Applied Sport Science Research, Queensland Academy of \\ Sport \\ E-mail: a.abbosh@uq.edu.au
}

\section{Introduction}

Recent advances in technology have influenced and changed many aspects of our lives. As technology becomes smaller in size, readily available and more efficient in operation, it can be used in increased aspects of our daily lives - competitive and recreational sports are no exception. The use of mobile sensor platforms to monitor and quantify aspects of an athlete's performance is well reported $[1,2]$. Using wireless technology, it is now possible to broadcast, store and analyse this data in real time.

The athletic environment is considered generally an electrically harsh environment and the aquatic environment is no exception where there are considerable challenges for the wireless transmission of athlete data. Of particular interest is the challenge of continuous data communications in the aquatic and mixed dry free-space/aquatic environment. Radio frequency transmission in and around water is particularly challenging as water is a highly attenuating media.

It is well known that while the $2.4 \mathrm{GHz}$ frequency or any higher band is convenient for high data rate applications, it is not suitable for underwater applications, though where there is periodic exposure to the air; burst mode transmission may be possible. Lower ISM bands (e.g. $433 \mathrm{MHz}$ ) are known to be less susceptible to the negative effects of water, though the size of antennas designed in these bands may preclude wearable applications.

Reviewing the literature shows that there are many types of configurations that can be utilized to build UHF antennas [3-6]. However, the majority of those configurations rely on a resonant structure, which is not suitable for the aquatic monitoring applications. This is because of the wide change in the operating environments which eventually causes a huge shift in the value of the resonant frequency. On the other hand and due to the increased interest in wideband applications, there are other types of antennas that can be modified for the aquatic environment, such as the corrugated tapered slot antenna $[7,8]$.

This paper addresses the challenge of designing a compact UHF antenna for aquatic monitoring systems. In the intended applications, the antenna is to be fixed on different parts of the athlete's body, thus it should be compact in size to avoid any obstruction to the athlete's normal performance. Designing an antenna 
that operates at $433 \mathrm{MHz}$ underwater and having a compact size might not be a very challenging task as the size is scaled by the high dielectric constant of the water. However, the challenge is when trying to use the same antenna to operate effectively not only underwater, but also above the water, such as when part of the athlete's body becomes above the water. In conclusion, the antenna designed for swimmers' monitoring system should achieve the following tasks: Firstly, it should operate at the UHF band assigned for the wireless monitoring system; secondly, it should have a compact size and thirdly, it should operate efficiently under and above the water with acceptable radiation efficiency.

\section{Design}

It is clear from the above discussions that a resonant antenna could not be the solution to the current task as the frequency of resonance shifts widely when the operating environment changes. The solution proposed in this paper is a mixture of double tapered slot structure coupled with array of slots that can be effective under different environments.

The structure for the proposed antenna is shown in Fig. 1. It has dual main tapered slots that feed array of slots extending along the two main directions of radiation. The dual tapered slots are used to achieve an omnidirectional radiation. The antenna is fed via a microstrip feeder, which makes the integration with the RF circuitry easy.

When the antenna is entirely underwater, the effective dielectric constant of the antenna's structure is highly affected by the high value of the dielectric constant of water, which is around 80 . However, when the antenna is above the water, the effective dielectric constant is much lower as the dielectric constant of the environment in this case (free-space) is close to 1 . In order to minimize the variation in value of the effective dielectric constant, superstrates, which are made of a similar material to that of the main substrate, are used to cover the two sides of the antenna forming a three-layered sandwiched structure. With this structure, the wide variation of the dielectric constant of the two operating environments of the antenna is minimized to a small variation in the effective dielectric constant as its value in this case is the result of effects of the main substrate, the two superstrates and the environment in which the antenna operates.

The initial dimensions of the antenna $(l \& w)$ are chosen to be equal to half and quarter of the effective wavelength, respectively, assuming that the antenna is underwater. The final dimensions are found using the optimization capabilities of the software CST Microwave Studio. The optimization was carried out in three different environments; antenna entirely immersed in water, partially immersed and finally in free-space. Effect of the human body for a sacral mounted antenna is also included in the optimization and simulations by including a model that represents the electrical characteristics of the different human tissues. The detailed dimensions of the antenna assuming the use of Rogers RT6010 (with dielectric constant $=10.2$, thickness of each layer $=1.27 \mathrm{~mm}$ ) as substrate and superstrates are: $w=40 \mathrm{~mm}, l=70 \mathrm{~mm}, s=1.3 \mathrm{~mm}, d=3.5 \mathrm{~mm}$. The microstrip feeder has a width of $0.7 \mathrm{~mm}$. 


\section{Results and Discussions}

The structure was simulated using the full-wave electromagnetic simulator CST Microwave Studio. Different operating environments were considered in the simulations, such as underwater, partially underwater, and above the water.

The results concerning the reflection coefficient is shown in Fig. 2. It is clear that the antenna has a return loss which is larger than $10 \mathrm{~dB}$ at the required operating frequency $(433 \mathrm{MHz})$. The antenna is supposed to have omnidirectional properties as the athlete may move in any situation and the data link should operate under any position for the athlete. The simulated radiation patterns of the antenna at $433 \mathrm{MHz}$ and three different operating environments are shown in Fig.3. It is obvious that the antenna achieves the required omnidirectional performance.

In order to make sure of the antenna's performance, the software was used to calculate the radiation efficiency under different operating environments. The results indicated that the efficiency is always larger than $60 \%$ at $433 \mathrm{MHz}$ when the antenna is either partially or entirely underwater. However, the efficiency is at around $40 \%$ when the antenna is above water because of the very small electrical size of the antenna when operating in free-space. It is to be noted here that the low efficiency of the antenna when operating in free-space environment is not of great concern as the wireless link in this case is free from the severe water attenuation.

The simulated results reveal that despite the compact size and harsh operating environment, the designed antenna can be used to efficiently establish a wireless link for aquatic monitoring system. The next step is to manufacture the antenna and test its performance. The measured results will be presented in the conference.

\section{References}

[1] N. Davey, M. Anderson, and D. James, "Validation trial of an accelerometer-based sensor platform for swimming," Sports Technology, vol. 1, no. 4, pp. 202-207, 2008.

[2] A. Wixted, D. Thiel, A. Hahn, C. Gore, D. Pyne, and D. James, "Measurement of Energy Expenditure in Elite Athletes using MEMS based inertial sensors," Journal IEEE Sensors, vol 7, no. 4, pp. 481-8, 2007.

[3] C. Balanis, Antenna theory; Analysis and design, $3^{\text {rd }}$ Edition, John Wiley, 2005.

[4] D. Psychoudakis, C. Chen, and J. Volakis, "Optimizing wearable UHF antennas for on-body operation," IEEE APS, pp. 4184 - 4187, 2007.

[5] D. Casciola, G. Miers, and R. Surette, "UHF antenna choices," IEEE Trans. Broadcasting, vol. 45, no. 1, pp. 93 - 105, 1999.

[6] R. Waterhouse, Printed Antennas for Wireless Communications, John Wiley, 2007.

[7] A. Abbosh, "Miniaturization of planar ultrawideband antenna via corrugation," IEEE Antennas and Wireless Propagation Letters, vol. 7, pp. 685 - 688, 2008.

[8] A. Abbosh, "Miniaturized microstrip-fed tapered-slot antenna with ultrawideband performance," IEEE Antennas and Wireless Propagation Letters, vol. 8, pp. $690-$ 692, 2009. 


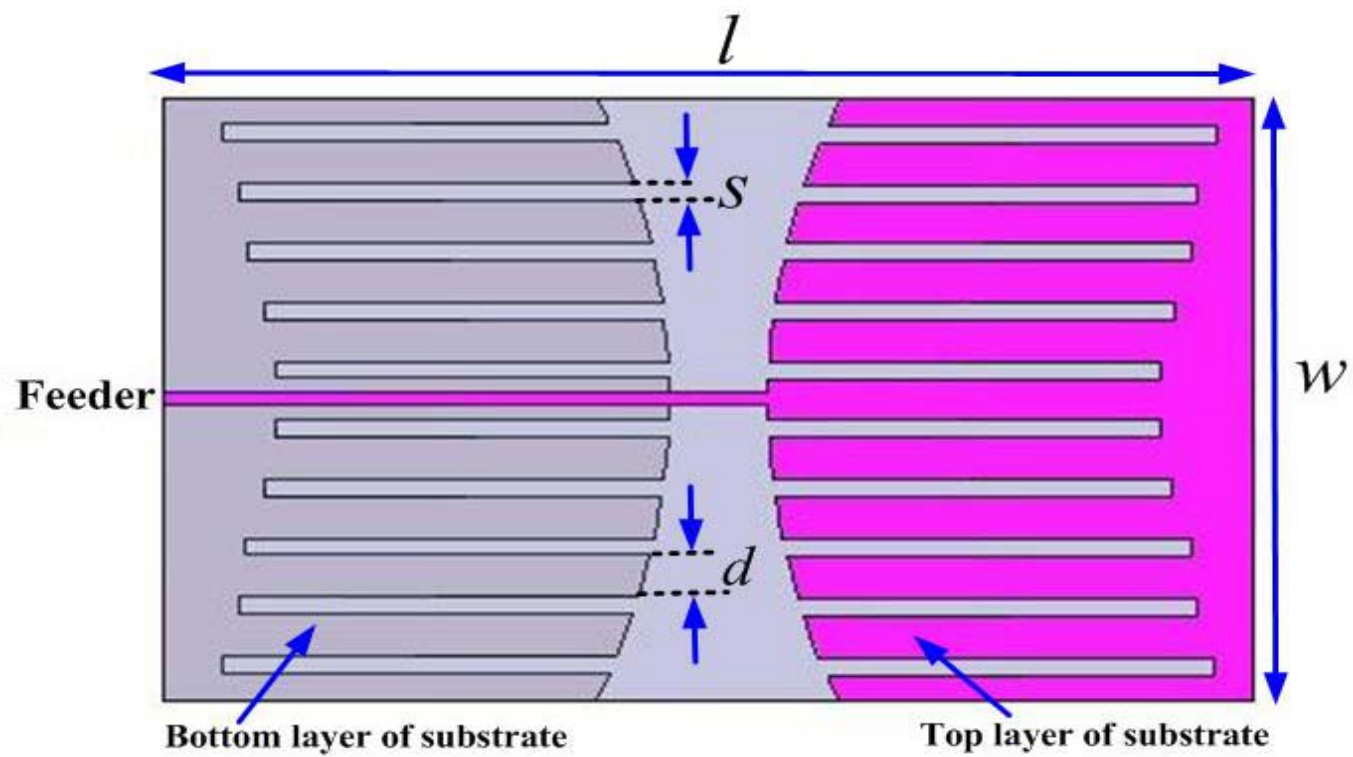

Fig.1 Configuration of the proposed antenna

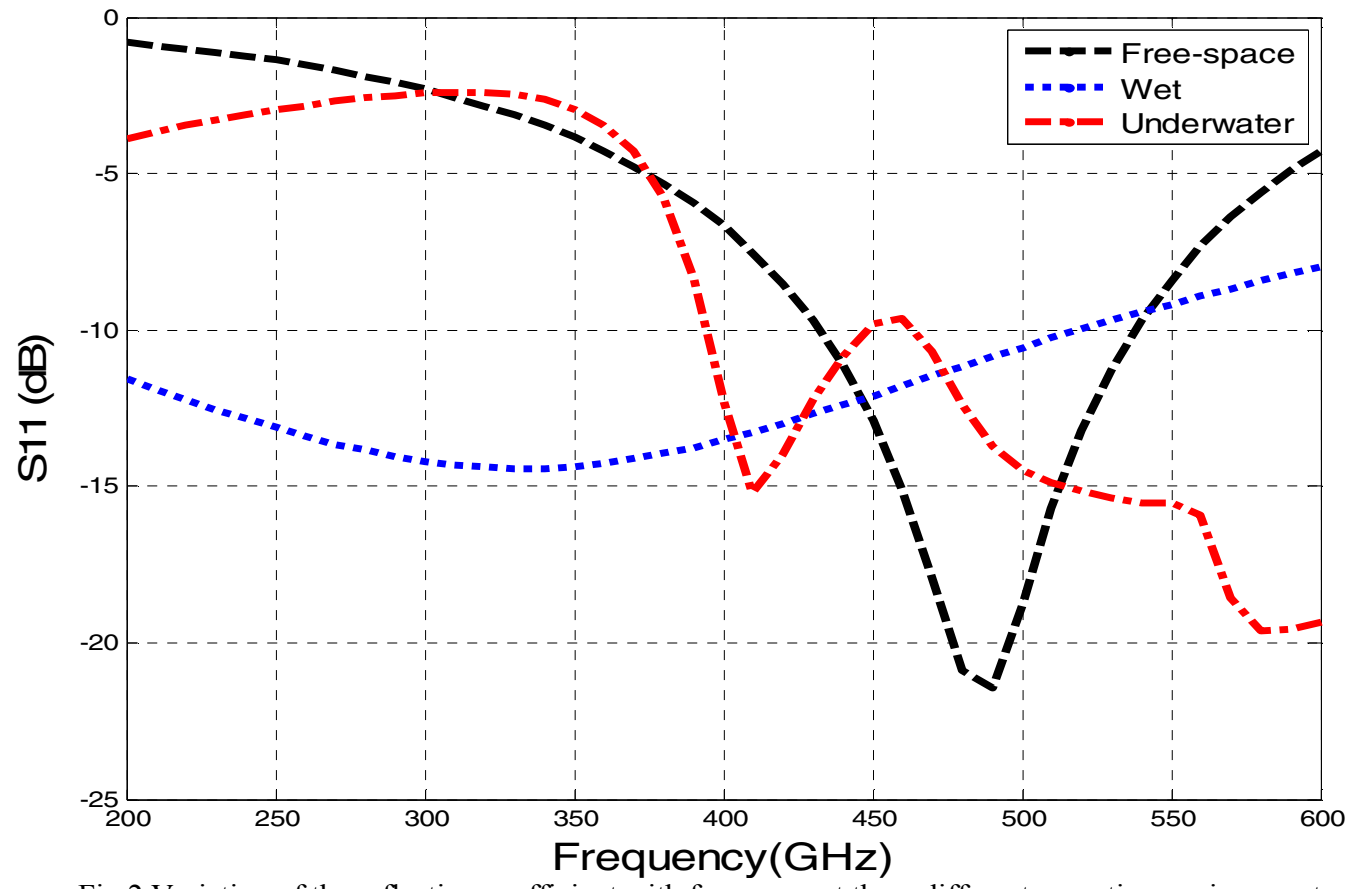

Fig.2 Variation of the reflection coefficient with frequency at three different operating environments.

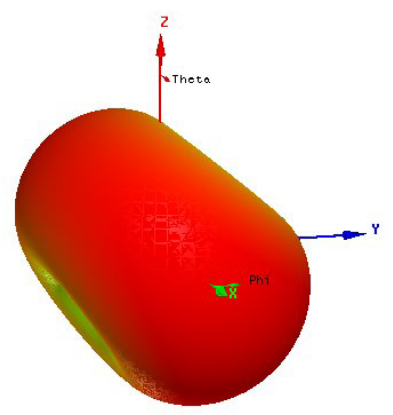

(a) Free-space

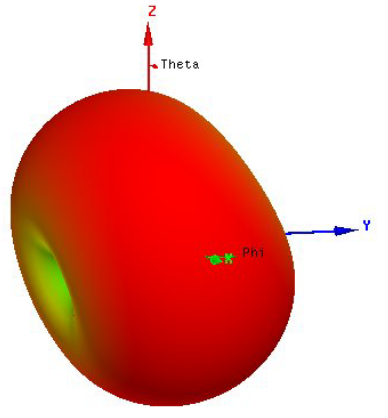

(b) Free-space/ water interface

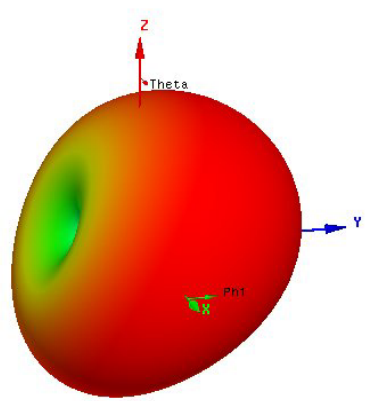

(c) Underwater

Fig. 3 The three-dimensional radiation pattern of the antenna at $433 \mathrm{MHz}$ 